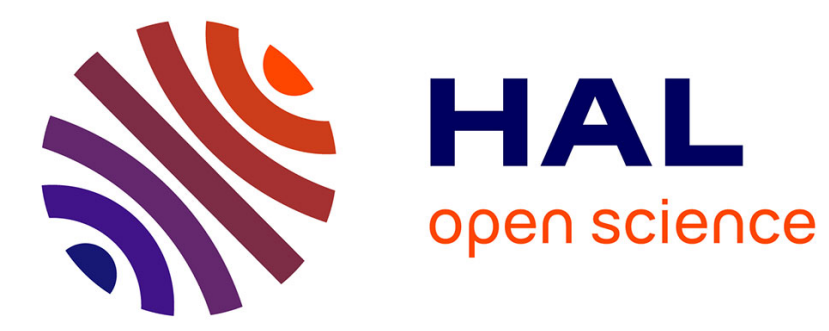

\title{
A simple three-wave Approximate Riemann Solver for the Saint-Venant-Exner equations
}

Emmanuel Audusse, Christophe Chalons, Philippe Ung

\section{To cite this version:}

Emmanuel Audusse, Christophe Chalons, Philippe Ung. A simple three-wave Approximate Riemann Solver for the Saint-Venant-Exner equations. 2016. hal-01204754v2

\section{HAL Id: hal-01204754 \\ https://hal.science/hal-01204754v2}

Preprint submitted on 12 Aug 2016

HAL is a multi-disciplinary open access archive for the deposit and dissemination of scientific research documents, whether they are published or not. The documents may come from teaching and research institutions in France or abroad, or from public or private research centers.
L'archive ouverte pluridisciplinaire HAL, est destinée au dépôt et à la diffusion de documents scientifiques de niveau recherche, publiés ou non, émanant des établissements d'enseignement et de recherche français ou étrangers, des laboratoires publics ou privés. 


\title{
A Simple three-WAVe Approximate Riemann Solver FOR THE SAINT-VENANT-EXNER EQUATIONS
}

\author{
E. Audusse* \\ C. Chalons ${ }^{\dagger}$ \\ P. Ung $\ddagger$
}

August 11, 2016

\begin{abstract}
Erosion and sediments transport processes have a great impact on industrial structures and on water quality. Despite its limitations, the Saint-Venant-Exner system is still (and for sure for some years) widely used in industrial codes to model the bedload sediment transport. In practice, its numerical resolution is mostly handled by a splitting technic that allows a weak coupling between hydraulic and morphodynamic distinct softwares but may suffer from important stability issues. In recent works, many authors proposed alternative methods based on a strong coupling that cure this problem but are not so trivial to implement in an industrial context. In this work, we then pursue two objectives. First we propose a very simple scheme based on an approximate Riemann solver, respecting the strong coupling framework, and we demonstrate its stability and accuracy through a number of numerical test cases. But, second, we reinterpret our scheme as a splitting technic and we extend the purpose to propose what should be the minimal coupling that ensures the stability of the global numerical process in industrial codes, at least when dealing with collocated finite volume method. The resulting splitting method is, up to our knowledge, the only one for which stability properties are fully demonstrated.
\end{abstract}

Key words. shallow-water equations, Exner equation, splitting method, finite volume method, positivity preserving, well-balanced scheme, approximate Riemann solver.

\section{Introduction.}

Sediment transport processes are usually divided into two main phenomena : suspended and bedload transport [48, 42]. To model bedload sediment transport phenomena that occur in large time and space scales in river hydraulics or coastal studies, one of the most commonly used models in engineering context $[35,34]$ is the Saint-Venant-Exner system, referred to as SWExner in the following. It takes the form of a system of three equations where the first two ones are nothing but the shallow water equations with topography and friction source terms whereas the last equation is

*LAGa, UMR CNRS 7539, Université Paris XiII, Sorbonne Paris Cité, F-93430 Villetaneuse, France - InRIA, Ange Project-Team, Rocquencourt, F-78153 Le Chesnay Cedex, France Cerema, Ange Project-Team, F-60280 Margny-Lès-Compiègne, France - LJlL, UMR CNRS 7958 , UPMC Université Paris VI, ANGE Project-Team, F-75005 Paris, France; http://www.math.univparis13.fr/ audusse/index.html, (audusse@math.univ-paris13.fr).

${ }^{\dagger}$ LMV, UMR CNRS 8100, Université de Versailles-Saint-Quentin-en-Yvelines, F-78035 Versailles Cedex, France; http://chalons.perso.math.cnrs.fr/, (christophe.chalons@uvsq.fr).

†MAPMO, UMR CNRS 7349, Université D'Orléans, F-45067 Orléans Cedex 2, France - INRIA, Ange Project-Team, Rocquencourt, F-78153 Le Chesnay Cedex, France - Cerema, Ange ProjectTeam, F-60280 Margny-Lès-Compiègne, France - LJll, UMR CNRS 7958, UPMC Université Paris Vi, ANGE Project-Team, F-75005 Paris, France; (philippe.ung@math.cnrs.fr). 
a simple conservation law that refers to the evolution in time of the topography due to the action of the fluid. Eventually the full system writes,

$$
\left\{\begin{array}{l}
\partial_{t} h+\partial_{x} h u=0, \\
\partial_{t} h u+\partial_{x}\left(h u^{2}+\frac{g h^{2}}{2}\right)=-g h\left(\partial_{x} b+T_{f}\right), \\
(1-\Phi) \partial_{t} b+\partial_{x} q_{s}=0,
\end{array}\right.
$$

where the unknowns are the water height $h(t, x)$, the flow velocity $u(t, x)$ and the bottom topography $b(t, x)$. The parameters are the gravitational acceleration $g$ and the porosity of the sediment layer $\Phi$ that will be set to zero hereafter. We also introduce the fluid discharge $q(t, x):=h(t, x) u(t, x)$. The friction term $T_{f}$ and the sediment flux $q_{s}$ are fundamental ingredients of the model since they ensure the coupling between the fluid and the solid parts. They are defined by semi-empirical formulae discussed in the next Section.

Both the separation between two distinct types of transport and the SWExner model itself present important limitations and drawbacks that have been discussed in a serie of recent papers $[39,1,12,22,23,19]$ where the authors exhibit different limitations of the classical SWExner model and introduce some improvements by different ways. In this work, we do not discuss this point and focus on the numerical strategy to compute approximate solutions of system (1).

The SWExner system (1) is a system of nonlinear conservation laws with source term that has been proved to be of hyperbolic type for some classical choices of sediment flux $q_{s}$ formula, see [13]. It is then natural to compute approximate solutions by using finite volume methods [31, 7] for their inherent properties of conservation and their ability to handle the discontinuous solutions. In this framework, the numerical method has to satisfy classical consistency and nonlinear stability (positivity, entropy, equilibria...) properties.

But the SWExner system (1) can also be viewed - it was indeed introduced in this spirit in the pioneering work of Exner [16] - as a coupling between two distinct systems in a "fluid-structure interaction" approach : on the one hand a fluid system, the classical Saint-Venant system (1a)-(1b), and on the other hand a conservation equation for the mass of sediment (1c). This approach is very attractive since it allows to use classical fluid solvers coupled to an external "structure" solver - that is in the simplest case reduced to a single conservation law. That is why it is often used for industrial studies $[35,34,32,33]$ : the core of these software suites is the resolution of hydraulic problems and to take into account the sedimentary aspect, they call sediment modules that are able to solve the Exner equation. This approach is not intrusive since the two modulus can run with different numerical parameters such as the time step, the space step or the numerical method and only communicate by exchanging particular data at some given times. It also allows to complexify the "structure" equation without modifying the fluid solver. But in this case, consistency and stability of both solvers is not sufficient to ensure the whole stability of the method and particular attention has to be paid to the way the interaction processes are performed.

Our main objective in this work is to perform a detailed study of these two different approaches and, more particularly, to use knowledge from the first approach, referred in the following to as the one-step approach, to propose stable interaction processes for the second approach, referred in the following to as the splitting approach. More precisely, we define and analyze a new one-step solver that we interpret in a second stage as a stable splitting method and then generalize to a unified splitting approach. Comparative studies of both one-step and splitting approaches, sometimes called coupled and uncoupled approaches, have been performed in the last decade by several authors $[10,29,28,13,2,45]$. They mostly conclude to the bad behavior of the splitting approach and then give rise to several works where the authors investigate one-step approaches $[44,15,5,9,36,46]$ by extending finite volume solvers developed in the early 2000's for the classical SW system to the 
SWExner system. We would like to emphasize that, in fact, they only conclude to the bad behavior of one possible way to perform the splitting approach, not of the splitting approach itself. First attempts to introduce stable splitting approaches were performed recently in $[43,45,30]$ where the authors propose what they call a weak coupling approach for the SWExner system by introducing a slight modification in the discretization of the Exner equation (1c) and in [26] where the authors deal with staggered grids.

The outline of the paper is as follows. In Section 2, we give some details on the SWExner system (1). In Section 3, we consider the one-step approach and we propose and analyze a positive and well-balanced three-wave Approximate Riemann Solver (denoted ARS in the following) that extends the well-balanced and positive solver introduced in [3] for the classical SW system. In Section 4, a serie of numerical test cases illustrates the performance of the scheme. In Section 5, the ARS scheme is then interpreted as a splitting approach where a slight modification is introduced in the fluid solver when compared to the classical (and then unstable) splitting approach. We then introduce the same modification in the strategy that is used in the industrial software TELEMAC and show that the method is now stable.

\section{Description of the mathematical model.}

The SWExner system (1) has to be closed by giving the friction term $T_{f}$ and the sediment flux $q_{s}$ in terms of the variables of the system $(h, h u, b)$. This is done in the first part of this Section. Then we give some properties of the SWExner model that will guide the derivation of the numerical solver.

\subsection{Closure formula.}

The friction term $T_{f}$ is usually defined by semi-empirical formulae proposed by hydraulic engineers in the late nineteenth or early twentieth centuries. In general, it only depends on the fluid quantities. One of the most popular is the Manning-Strickler formula,

$$
T_{f}=\frac{|q| q}{K_{s}^{2} h^{2} R_{h}^{4 / 3}},
$$

with $K_{s}$ the Strickler coefficient and $R_{h}$ the hydraulic radius such that,

$$
R_{h}=\frac{A}{P}
$$

where $A$ is the cross sectional area of the flow and $P$ corresponds to the wetted perimeter. When a rectangular channel is considered, this radius is then given by,

$$
R_{h}=\frac{l h}{l+2 h}
$$

where $l$ is the width of the channel. In the asymptotic regime where the water height is negligible compared to the channel width, the hydraulic radius then reduces to the water height : $R_{h} \sim h$. As previously mentioned, this term models amongst other things the action of the solid part on the fluid which is not related to the gravity; the latter is taken into account by the term of topography. Thus, this term includes the action of the rugosity at the microscopic scale which do not explicitly appear in the term of topography. It is therefore a fundamental ingredient of the coupled model. Let us make precise that this term can be defined by different formulae. In particular in [24], the friction term derives from a wall law at the level of the Navier Stokes model. Note also that it will still be neglected in some of the numerical tests to compare the results of the proposed scheme with those of 
the existing literature.

The sediment flux formula $q_{s}$ is also computed by semi-empirical formulae and is often a function of the fluid quantities $(h, u)$ only. The number of formulae proposed in the literature is huge and is still a research area in the hydraulic community. The range of applications of any of them is far from being universal, depending on the nature of the fluid flow and the characteristics of the solid bed. One can exhibit two main categories depending on whether a threshold value is used or not for the sediment transport to start. We will use in the numerical applications the Grass [25] formula,

$$
q_{s}(t, x)=A_{g}|u|^{m-1} u,
$$

and the Meyer-Peter-Müller formula,

$$
q_{s}(t, x)=A_{m}\left(\tau^{\star}-\tau_{c}^{\star}\right)^{3 / 2},
$$

where $\tau^{\star}$ refers to a non-dimensional friction term that is weighted by the buoyancy of the sediment particles,

$$
\tau^{\star}=\frac{\rho_{w} h T_{f}}{\left(\rho_{s}-\rho_{w}\right) d},
$$

that is known as the Shields parameter, where $\rho_{w}, \rho_{s}$ are the density of the water and solid phase respectively and $d$ the mean granular diameter. Note that some authors recently introduced formulae that explicitly depend on the sediment depth $b[18]$.

Let us note that we will perform numerical test cases using the above formula but the theoretical part of our paper works for very general form of the friction coefficient and sediment flux as soon as they satisfy general properties that will be precised hereafter.

\subsection{Properties of the model.}

The hyperbolicity of the SWExner model (1) has been studied in [13]. The authors showed that for a large class of classical closure formula, the system is hyperbolic. They also exhibit an interesting property : unlike the classical SW system, the product of the eigenvalues of the SWExner system is always negative. This property will be used in the last part of this paper to derive a stable splitting approach. In the following, we always consider that the SWExner system is hyperbolic.

Like the classical shallow water model (with fixed topography), some important stability properties have to be satisfied by a numerical method to correctly approximate the solutions of SWExner system (1). In particular, the numerical scheme has to ensure the positivity of the water height and the wellbalanced property characterized here by the preservation of two equilibria,

- the lake at rest equilibrium defined by,

$$
\partial_{x}(h+b)=0, \quad u=0,
$$

- the constant slope equilibrium defined by,

$$
\partial_{x} h=\partial_{x} u=\partial_{x x} b=0, \quad \partial_{x} b+T_{f}=0 .
$$

Note that contrary to the case of the classical SW system, the lake at rest equilibrium (2.4) is the unique steady state over non constant slope that is admitted by the SWExner system, at least for solid discharge defined by formulae without threshold such as the Grass one (2.2). Let us remark that the validity of the well-balanced property is of great interest when erosion near a steady state is considered. It is the case for the advection of a dune at low Froude number or for Newton or Soni 
experiments [37, 47]. A third stability criteria is the existence of an energy inequality. Even for the simpler case of the classical SW system, the way to ensure a fully discrete energy inequality is not obvious, see [7]. It is then much more tricky for the SWExner model where issues already occur at the continuous level, see $[49,19]$. A last important point is to be able to identify the behavior of the numerical scheme when the sediment transport vanishes since we would like to recover a relevant scheme for the classical SW model.

\section{A one-step numerical scheme.}

In this Section, we consider a Godunov-type finite volume numerical method introduced in [3] for the discretization of the classical SW system and we extend it to the new framework of the SWExner system (1). This method is based on an Approximate Riemann Solver (ARS in what follows). We briefly recall the notations and we detail its extension, i.e. the way to update the bottom quantities and the wave speeds. We then give some properties of the discrete solver. Note that the friction term is computed in a second step that is briefly detailed at the end of this section.

\subsection{Definition of the Approximate Riemann Solver.}

A Godunov-type finite volume approximation of SWExner system (1) is based on the computation of a piecewise constant approximation of the solution on a given partition of the domain by using the solution of a Riemann problem at each interface of the mesh [7,31]. An ARS solver then replaces the solution of the exact Riemann problem - that can be very complex for systems - by an approximate description of the solution. In [3], we introduced a three wave ARS for the classical SW system. Its extension to the SWExner system can be described with obvious notation by,

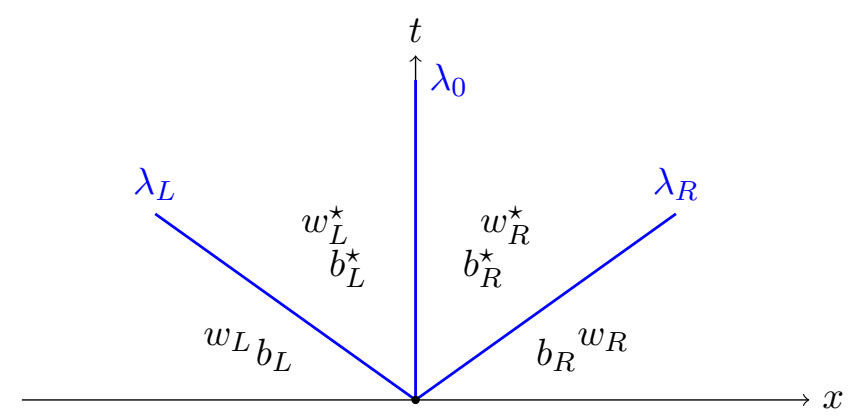

$$
\lambda_{L} \leqslant 0 \leqslant \lambda_{R}
$$

Figure 1 - Local Riemann problem.

The key point is now the definition of

- the intermediate states $w_{L, R}^{\star}$ and $b_{L, R}^{\star}$ where $w^{\star} \in \mathbb{R}_{+} \times \mathbb{R}$ stands for the fluid quantities $(h, h u)$ and $b^{\star}$ for the solid sediment depth,

- the wave speeds $\lambda_{L}$ and $\lambda_{R}$ for which we impose (3.1). Unlike the classical SW system, it is not a restriction for the study of the SWExner system since it is proved in [13] that it is always satisfied for the solution of the exact Riemann problem.

Let first suppose the wave speeds $\lambda_{L}$ and $\lambda_{R}$ are known. The definition of the intermediate states denoted by $\star$ needs for the definition of six scalar values. Three of them are given by the so-called consistency relations that are needed to prove that the ARS is consistent in the integral 
sense $[20,21,27]$ with (1), namely,

$$
\left\{\begin{aligned}
h_{R} u_{R}-h_{L} u_{L}=\lambda_{L}\left(h_{L}^{\star}-h_{L}\right)+\lambda_{R}\left(h_{R}-h_{R}^{\star}\right), \\
\left(h_{R} u_{R}^{2}+\frac{g h_{R}^{2}}{2}\right)-\left(h_{L} u_{L}^{2}+\frac{g h_{L}^{2}}{2}\right)+g \Delta x\left\{h \partial_{x} b\right\} \\
=\lambda_{L}\left(h_{L}^{\star} u_{L}^{\star}-h_{L} u_{L}\right)+\lambda_{R}\left(h_{R} u_{R}-h_{R}^{\star} u_{R}^{\star}\right), \\
q_{s}\left(w_{R}\right)-q_{s}\left(w_{L}\right)=\lambda_{L}\left(b_{L}^{\star}-b_{L}\right)+\lambda_{R}\left(b_{R}-b_{R}^{\star}\right),
\end{aligned}\right.
$$

where $\left\{h \partial_{x} b\right\}$ denotes a consistent approximation of the source term $(0,-g h \Delta b, 0)^{T}$. It remains to define three relations. Two of them were introduced in [3] to ensure the preservation of the lake at rest equilibrium (2.4),

$$
\left\{\begin{array}{l}
h_{L}^{\star}+b_{L}^{\star}=h_{R}^{\star}+b_{R}^{\star}, \\
h_{L}^{\star} u_{L}^{\star}=h_{R}^{\star} u_{R}^{\star} .
\end{array}\right.
$$

Combined with the first two relations (3.2) and (3.3) they defined in [3] a complete ARS solver for the classical SW system. The last relation is based on a minimization problem on the energy of displacement of the sediment part under the consistency constraint (3.4),

$$
\begin{aligned}
\min \mathcal{F}\left(b_{L}^{\star}, b_{R}^{\star}\right) & =\left(\left\|b_{L}-b_{L}^{\star}\right\|^{2}+\left\|b_{R}-b_{R}^{\star}\right\|^{2}\right) \\
\text { u.c. } \mathcal{H}\left(b_{L}^{\star}, b_{R}^{\star}\right) & =\lambda_{L}\left(b_{L}^{\star}-b_{L}\right)+\lambda_{R}\left(b_{R}-b_{R}^{\star}\right)-\left(q_{s}\left(w_{R}\right)-q_{s}\left(w_{L}\right)\right)=0 .
\end{aligned}
$$

Note that this energy argument was already introduced in [8] for the same problem, but in a different framework. Note also we can in addition introduce an energy of deformation in order to penalize the high slope of the interface between sediment and water.

Once the nonlinear system (3.2)-(3.7) is solved - see next subsection, the quantities are updated by taking the mean value of the solution of the ARS on each cell of the mesh. The stability of the whole solver is ensured under the classical CFL restriction on the time step which ensures that two Riemann problems do not interact,

$$
\Delta t<\frac{\Delta x}{2 \max \left(\left|\lambda_{L}\right|, \lambda_{R}\right)}
$$

where the function max is taken over the set of all considered Riemann problems set at each interface $x_{i+1 / 2}$.

\subsection{Expression of the intermediate states.}

The solution to the minimization problem (3.7) can be computed independently. It leads to the following expressions of the intermediate states $b^{\star}$,

$$
\begin{aligned}
& b_{L}^{\star}=b_{L}+\frac{\lambda_{L}}{\lambda_{L}^{2}+\lambda_{R}^{2}} \Delta q_{s}, \\
& b_{R}^{\star}=b_{R}-\frac{\lambda_{R}}{\lambda_{L}^{2}+\lambda_{R}^{2}} \Delta q_{s} .
\end{aligned}
$$

Then easy calculations [3] lead to the explicit formulae for the water heights,

$$
\begin{aligned}
& h_{L}^{\star}=h_{H L L}+\frac{\lambda_{R}}{\lambda_{R}-\lambda_{L}} \Delta b^{\star}, \\
& h_{R}^{\star}=h_{H L L}+\frac{\lambda_{L}}{\lambda_{R}-\lambda_{L}} \Delta b^{\star},
\end{aligned}
$$


where $h_{H L L}$ is the intermediate water height of the well-known HLL Riemann solver [27],

$$
h_{H L L}=\frac{\lambda_{R} h_{R}-\lambda_{L} h_{L}}{\lambda_{R}-\lambda_{L}}-\frac{1}{\lambda_{R}-\lambda_{L}}\left(h_{R} u_{R}-h_{L} u_{L}\right)
$$

and for the value of the discharge,

$$
\begin{gathered}
q^{\star}:=h_{L}^{\star} u_{L}^{\star}=h_{R}^{\star} u_{R}^{\star}, \\
q^{\star}=q_{H L L}-\frac{g}{\lambda_{R}-\lambda_{L}} \Delta x\left\{h \partial_{x} b\right\},
\end{gathered}
$$

where again, $q_{H L L}$ corresponds to the intermediate discharge of the HLL Riemann solver [27], namely,

$$
q_{H L L}=\frac{\lambda_{R} h_{R} u_{R}-\lambda_{L} h_{L} u_{L}}{\lambda_{R}-\lambda_{L}}-\frac{\left(h_{R} u_{R}^{2}+\frac{g h_{R}^{2}}{2}\right)-\left(h_{L} u_{L}^{2}+\frac{g h_{L}^{2}}{2}\right)}{\lambda_{R}-\lambda_{L}} .
$$

Updating the solution is then just performed by a simple integration on each cell,

$v_{i}^{n+1}=\left(\frac{1}{2}-\left(\lambda_{R}\right)_{i-1 / 2}^{n} \frac{\Delta t^{n}}{\Delta x}\right)\left(v_{R}^{\star}\right)_{i-1 / 2}^{n}+\left(\left(\lambda_{R}\right)_{i-1 / 2}^{n}-\left(\lambda_{L}\right)_{i+1 / 2}^{n}\right) \frac{\Delta t^{n}}{\Delta x} v_{i}^{n}+\left(\frac{1}{2}+\left(\lambda_{L}\right)_{i+1 / 2}^{n} \frac{\Delta t^{n}}{\Delta x}\right)\left(v_{L}^{\star}\right)_{i+1 / 2}^{n}$,

for $v=(h, h u, b)$ and with notation,

$$
\left(w_{r}\right)_{i-1 / 2}^{n}=w_{r}\left(h_{i-1}^{n}, u_{i-1}^{n}, b_{i-1}^{n}, h_{i}^{n}, u_{i}^{n}, b_{i}^{n}\right) .
$$

\subsection{Approximation of the wave velocities $-\lambda_{L}$ and $\lambda_{R}$.}

It now remains to define the values of the wave velocities $\lambda_{L}$ and $\lambda_{R}$. Let us recall that for wellknown stability reasons [7,31], $\lambda_{L}$ and $\lambda_{R}$ must be upper bounds of the eigenvalues of the Jacobian matrix $A$ of the flux function of system (1). Easy calculations give

$$
A=\left[\begin{array}{ccc}
0 & 1 & 0 \\
g h-u^{2} & 2 u & g h \\
\tilde{\alpha} & \tilde{\beta} & 0
\end{array}\right], \quad \tilde{\alpha}=\frac{\partial q_{s}}{\partial h}, \quad \tilde{\beta}=\frac{\partial q_{s}}{\partial q} .
$$

The exact values of the characteristic speeds are given by the roots of the characteristic polynomial of $A$, namely,

$$
p_{A}(\lambda)=\lambda^{3}-2 u \lambda^{2}-\left(g h(1+\tilde{\beta})-u^{2}\right) \lambda-g h \tilde{\alpha}=0 .
$$

These roots can be computed analytically, at least for simple enough sediment flux formulae, by using Cardan's formulae [6]. But recall that we are only interested in upper bounds of these exact characteristic speeds, i.e. of the solutions of (3.16). Several strategies to estimate these upper bounds have been proposed in the literature but most of them are valid under strong assumptions on the polynomial coefficients. Upper bounds proposed by Nickalls [38] turn out to be accurate and can be applied to any polynomial with real roots. It consists in differentiating the polynomial until we obtain a quadratic polynomial, here,

$$
3 \lambda^{2}-4 u \lambda-\left(g h(1+\tilde{\beta})-u^{2}\right)=0 .
$$

The solutions $\lambda_{ \pm}$of the corresponding equation can be then divided into two parts $x_{0}$ and $\Omega$,

$$
\lambda_{ \pm}=x_{0} \pm \Omega, \quad x_{0}=\frac{2 u}{3}, \quad \Omega=\frac{1}{3} \sqrt{u^{2}+3 g h(1+\tilde{\beta})} .
$$

In this form, we can exhibit the abscissa of the inflexion point which is equal to $x_{0}$, and $\Omega$ can be interpreted as the distance in the horizontal direction between the two local extrema of the polynomial 
(3.16) and the inflexion point. The Nickalls' theorem states that all the roots of (3.16) lie in the range bounded by $x_{0} \pm 2 \Omega$. That is why, we define the wave velocities by,

$$
\begin{aligned}
& \lambda_{L}=x_{0}-2 \Omega \leq 0, \\
& \lambda_{R}=x_{0}+2 \Omega \geq 0,
\end{aligned}
$$

where the inequalities are a direct consequence of the fact that the product of the eigenvalues of matrix $A$ is negative, see [13]. Note that when sediment transport vanishes, i.e. when considering the classical SW model, the proposed characteristic speeds reduce to,

$$
\lambda_{L}=\frac{2}{3}\left(u-\sqrt{u^{2}+3 g h}\right), \quad \lambda_{R}=\frac{2}{3}\left(u+\sqrt{u^{2}+3 g h}\right) .
$$

Recalling the exact characteristic speeds for the classical SW system with topography source term are,

$$
\lambda_{ \pm}=u \pm \sqrt{g h}, \quad \lambda_{0}=0,
$$

we conclude the Nickalls' approximation is rather accurate in this case since,

$$
\frac{\max \left(\left|\lambda_{L}\right|,\left|\lambda_{R}\right|\right)}{\max \left(\left|\lambda_{ \pm}\right|\right)}=\frac{2}{3} \frac{|u|+\sqrt{u^{2}+3 g h}}{|u|+\sqrt{g h}} \in\left[1, \frac{4}{3}[,\right.
$$

where the minimum value is reached when the flow is transcritic, i.e. when

$$
\operatorname{Fr}=\frac{|u|}{\sqrt{g h}}=1 \text {. }
$$

In the numerical tests, we always use definitions (3.19)-(3.20) instead of the exact roots [6]. Note that the effective computation of Nickalls' approximations only depends on the derivative of the sediment flux as a function of the discharge. We retrieve a behavior that was exhibited in [2] in the study of the stability of a relaxation solver.

\subsection{Discretization of the friction term.}

It remains to define the numerical way to handle the friction term. This has been discussed in details in $[40,14]$. Here we choose to consider an implicit splitting method that is proposed in these works. Let us denote $q_{i}^{n+1}$ the solution of the proposed ARS at time $t^{n+1}$. Then, the solution of (1) $\tilde{q}_{i}^{n+1}$ at time $t^{n+1}$ is obtained by solving the equation,

$$
\tilde{q}_{i}^{n+1}=q_{i}^{n+1}-g \Delta t \frac{\left|\tilde{q}_{i}^{n+1}\right| \tilde{q}_{i}^{n+1}}{K_{s}^{2} h_{i}^{n+1}\left(R_{h, i}^{n+1}\right)^{4 / 3}} .
$$

By imposing in addition that $\tilde{q}_{i}^{n+1}$ and $q_{i}^{n+1}$ have the same sign, we obtain,

$$
\tilde{q}_{i}^{n+1}=\left\{\begin{array}{ll}
\frac{-1+\sqrt{1+4 a q_{i}^{n+1}}}{2 a}, & \text { if } q_{i}^{n+1}>0 \\
\frac{1-\sqrt{1-4 a q_{i}^{n+1}}}{2 a}, & \text { if } q_{i}^{n+1} \leq 0
\end{array}, \quad a=\frac{g \Delta t}{K_{s}^{2} h_{i}^{n+1}\left(R_{h, i}^{n+1}\right)^{4 / 3}} .\right.
$$

Note that a semi-implicit treatment of the source term is usually prescribed [14]. Our choice is motivated by the preservation of the constant slope equilibrium (2.5) since the semi-implicit treatment is possible at the price of a loss of the well-balanced property, see next section. Moreover the computation of the solution of relation (3.22) is explicit and therefore not costly. Note that an explicit treatment of the source term is also possible but is proved to be less stable, especially on wet-dry interfaces [14]. 


\subsection{Properties of the ARS}

In this subsection, we study two important properties of the numerical scheme,

- the positivity of the water depth,

- the well-balanced property for the lake at rest (2.4) and constant slope (2.5) equilibria.

Let us begin with the positivity of the water depth. In the context of ARS, this property reduces to the positivity of the intermediate water depths $h^{\star}$. As far as (3.10) and (3.11) are considered, this property is not satisfied. We thus propose a similar treatment to the one used in the case of the classical SW system [3] and we define the new intermediate states $\tilde{h}^{\star}$ with,

1. if $\Delta b^{\star} \geqslant 0$,

$$
\begin{aligned}
& \lambda_{R} \tilde{h}_{R}^{\star}=\lambda_{R} \max \left(h_{R}^{\star}, 0\right), \\
& \lambda_{L} \tilde{h}_{L}^{\star}=\lambda_{L} h_{L}^{\star}-\lambda_{R}\left(h_{R}^{\star}-\tilde{h}_{R}^{\star}\right),
\end{aligned}
$$

2. if $\Delta b^{\star}<0$,

$$
\begin{aligned}
& \lambda_{L} \tilde{h}_{L}^{\star}=\lambda_{L} \max \left(h_{L}^{\star}, 0\right), \\
& \lambda_{R} \tilde{h}_{R}^{\star}=\lambda_{R} h_{R}^{*}-\lambda_{L}\left(h_{L}^{\star}-\tilde{h}_{L}^{\star}\right) .
\end{aligned}
$$

We can easily check that these modifications preserve the positivity of the water heights. Indeed, for the first case, it is clear that $\tilde{h}_{R}^{\star}$ is positive. The value of $\tilde{h}_{R}^{\star}$ could be $h_{R}^{\star}$ or 0 . In the first situation, $\tilde{h}_{L}^{\star}=h_{L}^{\star}$ which is positive, and in the second one,

$$
\lambda_{L} \tilde{h}_{L}^{\star}=\lambda_{L} h_{L}^{\star}-\lambda_{R} h_{R}^{\star} \quad \Longleftrightarrow \quad h_{R}^{\star}=h_{L}^{\star}-\frac{\lambda_{R}}{\lambda_{L}} h_{R}^{\star}>0 .
$$

We apply the same calculations to prove the positivity of the water height in the case $\Delta b^{\star}<0$.

Let us now turn to the well-balanced property for the lake at rest equilibrium (2.4). Recall that we still have to define the discrete approximation of the source term $\left\{h \partial_{x} b\right\}$ in relation (3.3). As in [3] we propose the following definition,

$$
\left\{h \partial_{x} b\right\}= \begin{cases}\frac{h_{L}+h_{R}}{2 \Delta x} \min \left(h_{L}, \Delta b\right), & \text { if } \Delta b \geqslant 0, \\ \frac{h_{L}+h_{R}}{2 \Delta x} \max \left(-h_{R}, \Delta b\right), & \text { if } \Delta b<0 .\end{cases}
$$

Combined with relations (3.5)-(3.6), this definition obviously preserves the lake at rest steady state (2.4).

We finally consider the constant slope equilibrium (2.5). Starting from constant water depth and velocity, the discrete water depth remains obviously constant - the intermediate states $h^{*}$ are perturbed, but in a symmetric way. Second, it is easy to check that under the hypothesis of the constant slope equilibrium (2.5), the solution of the second degree equation (3.22) that satisfies $q_{i}^{n+1} q_{i}^{n} \geq 0$ is $q_{i}^{n+1}=q_{i}^{n}$. Note the fact that a splitting strategy is able to preserve a steady state is not so usual. It is due to the fact that when considering the constant slope equilibrium (2.5) hypothesis, the treatment of the source term can be written as a one step method since the water depth is constant in time. It is not the case for a semi-implicit treatment of the source term that hence is not able to preserve the steady state.

We conclude this subsection by noting that the solver obviously reduces to the classical ARS for the SW system introduced in [3] when the sediment flux $q_{s}$ vanishes, with the definition (3.21) for the wave velocities. 


\section{Numerical results.}

In order to validate the proposed ARS scheme, called SimSol (for "Simple Solver") in the Figures, we perform a series of classical test cases from the literature and we extend them to cover additional flow regimes. Thus we study the simulation of dune evolution in fluvial $[29,28,15,11,5,4]$, transcritical [13] and torrential [4] flow regimes, and the dam break problem over a moveable bed [36, 2] with or without dry state. Up to our knowledge, we propose the first complete study of these cases namely the flow over a bump and the dam break. For all test cases, we compare our results with those obtained for a scheme based on the computation of an approximate Jacobian matrix and so called Intermediate Field Capturing Riemann solver [41, 17] and another one based on a relaxation approach [2]. They are respectively referred as to IFCP and Relaxation thereafter. To complete the study, we determine the error curves by considering a reference solution computed with the IFCP scheme in a mesh with 4000 cells. For all test cases we consider the Grass formula (2.2) since it is widely used in the literature. In addition, and in order to compare with the results presented in the literature, we do not consider the friction term, except for the last test case, namely the dam break over dry bed.

\subsection{Dune evolution in a fluvial flow.}

This classical test case is a sediment transport problem which considers a bump under a fluvial flow. It is well known that the usual splitting strategy works well for this test case [13]. The length of the channel is $L=1000 \mathrm{~m}$ and the initial data are parametrized by,

$$
\begin{cases}b(0, x) & = \begin{cases}0.1+\sin ^{2}\left(\frac{(x-300) \pi}{200}\right), & \text { if } 300 \leqslant x \leqslant 500 m, \\ 0.1, & \text { elsewhere, }\end{cases} \\ h(0, x) & =10-b(0, x), \\ u(0, x) & =\frac{q_{0}}{h(0, x)},\end{cases}
$$

with $q(t, 0)=q_{0}=10 \mathrm{~m}^{2} / \mathrm{s}$ the inflow discharge. Fig. 2 shows the topography at time $T=700 \mathrm{~s}$ with a mesh of 2000 elements. It clearly appears that the relaxation method introduces a very high diffusive effect. The other two methods give very similar results in agreement with those in the literature. On fig. 3, it appears that the proposed ARS is first order accurate even if a sharp front appears at the front of the bump.
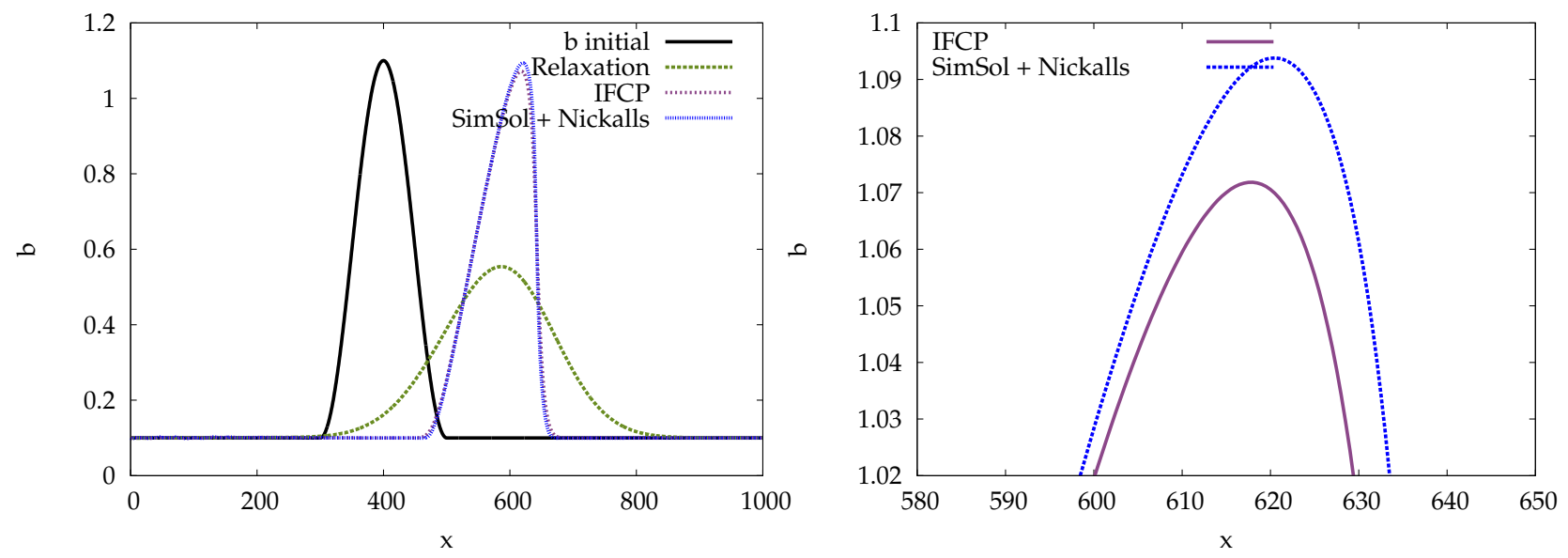

Figure 2 - Fluvial flow: Comparison of dune evolution for different numerical schemes. 


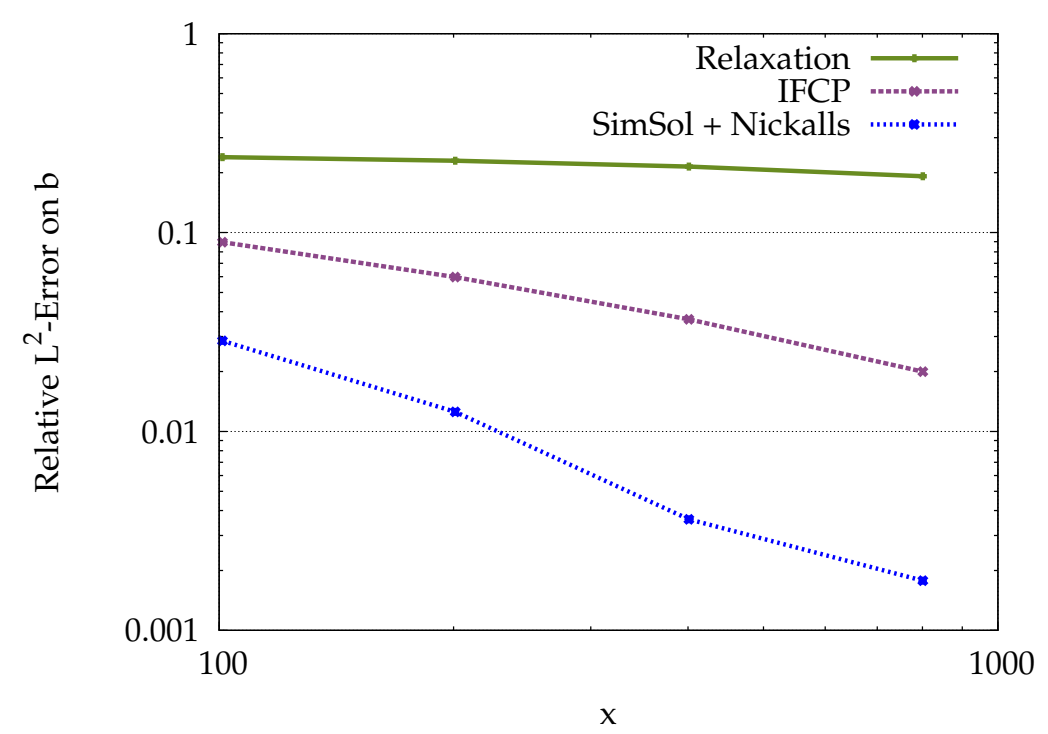

Figure 3 - Fluvial flow: Relative $L^{2}$-error on $b$.

\subsection{Dune (anti-dune) evolution in a torrential flow.}

This test case is a modified version of the one presented in [4]. A torrential flow over a moveable bed with an initial bump permits to deal with the anti-dune phenomenon. The channel is $L=24 \mathrm{~m}$ long and the initial bottom topography is,

$$
b(0, x)= \begin{cases}0.2-0.05(x-10)^{2}, & \text { if } 8 \leqslant x \leqslant 12 m, \\ 0, & \text { elsewhere }\end{cases}
$$

We impose a uniform discharge $q(0, x)=q_{0}=2 \mathrm{~m}^{2} / \mathrm{s}$ and evaluate the corresponding water height in the stationary state for the SW equations thanks to the Bernoulli's law,

$$
\left\{\begin{array}{l}
q(t, x)=q_{0}, \\
\frac{q_{0}^{2}}{2 g h^{2}}+h+b=H_{0}=\frac{q_{0}^{2}}{2 g h_{0}^{2}}+h_{0}+b(0,0),
\end{array}\right.
$$

with $h(t, 0)=h_{0}=0.5 \mathrm{~m}$. The Grass formula (2.2) defines the solid flux with parameters $A_{g}=0.001$ and $m=3$. We run the test case with a 2400-element mesh and consider the solution at time $T=\{6 s, 10 s, 15 s, 30 s, 50 s\}$. In this particular test case, the erosion operates after the top of the bump; at this point the velocity is greater than before the top of the dune causing the upstream migration of the anti-dune. The deposition of sediments occurs at the upstream of the bump. Here also the relaxation solver is very diffusive. On the contrary, both ARS and IFCP schemes compute well the shock formation at the front of the anti-dune, see fig. 4. In this test case, the schemes are not first order accurate, see fig. 5. This is due to the presence of the shock front before the anti-dune.

\subsection{Dune evolution in a transcritical flow without shock.}

This test case describes a transcritical flow without shock over a bump [13]. The length of the channel is $L=10 \mathrm{~m}$ and the initial data are defined by,

$$
\left\{\begin{array}{l}
b(0, x)=0.1+0.1 e^{-(x-5)^{2}} \\
h(0, x)=0.4-b(0, x) \\
q(0, x)=0.6
\end{array}\right.
$$



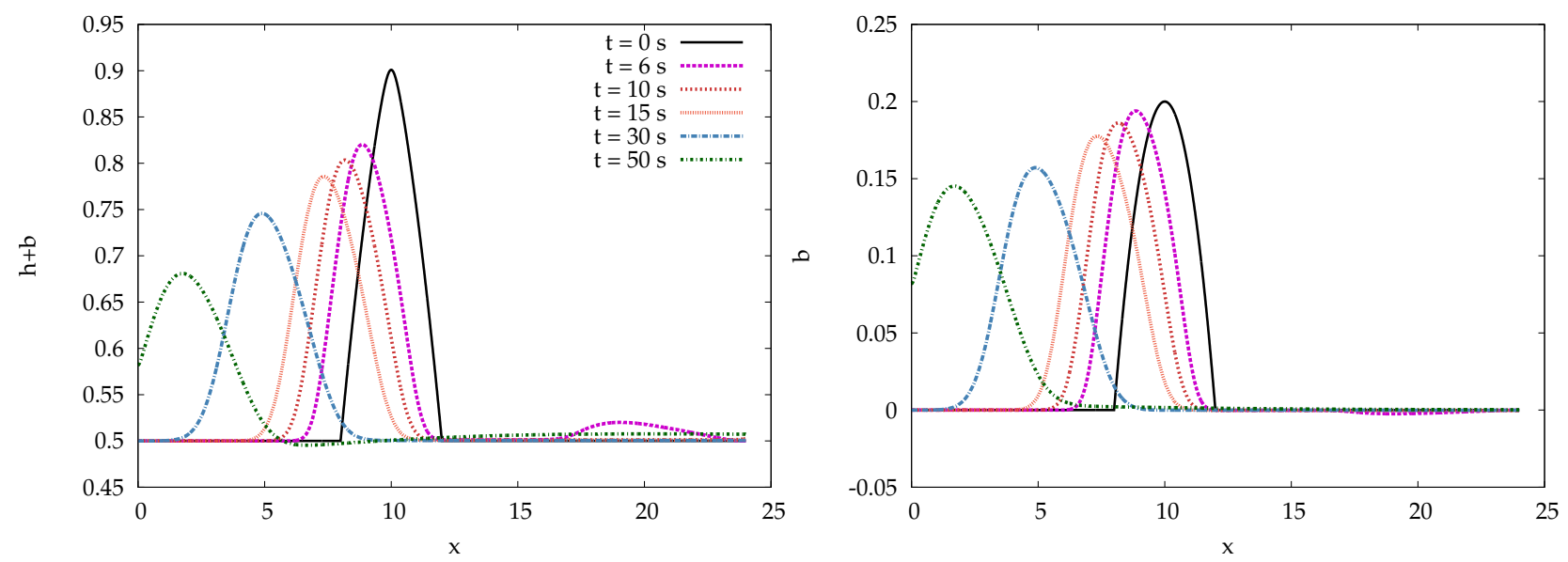

(a) Relaxation
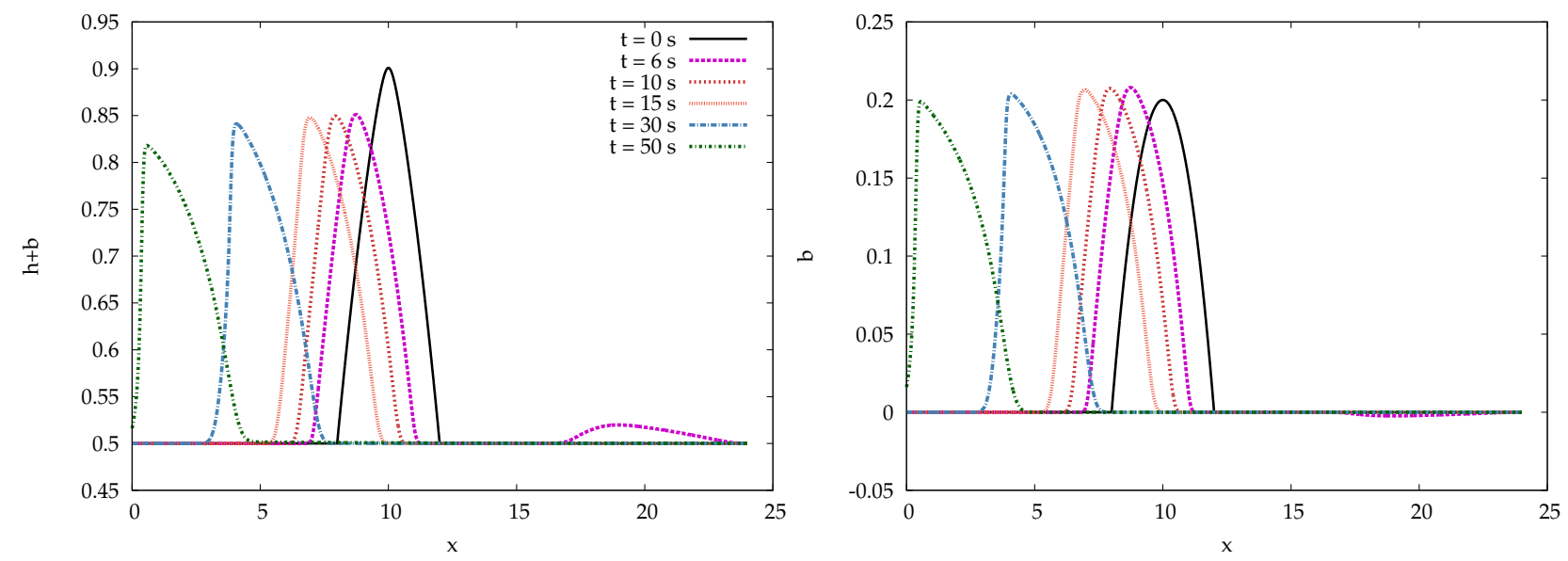

(b) IFCP
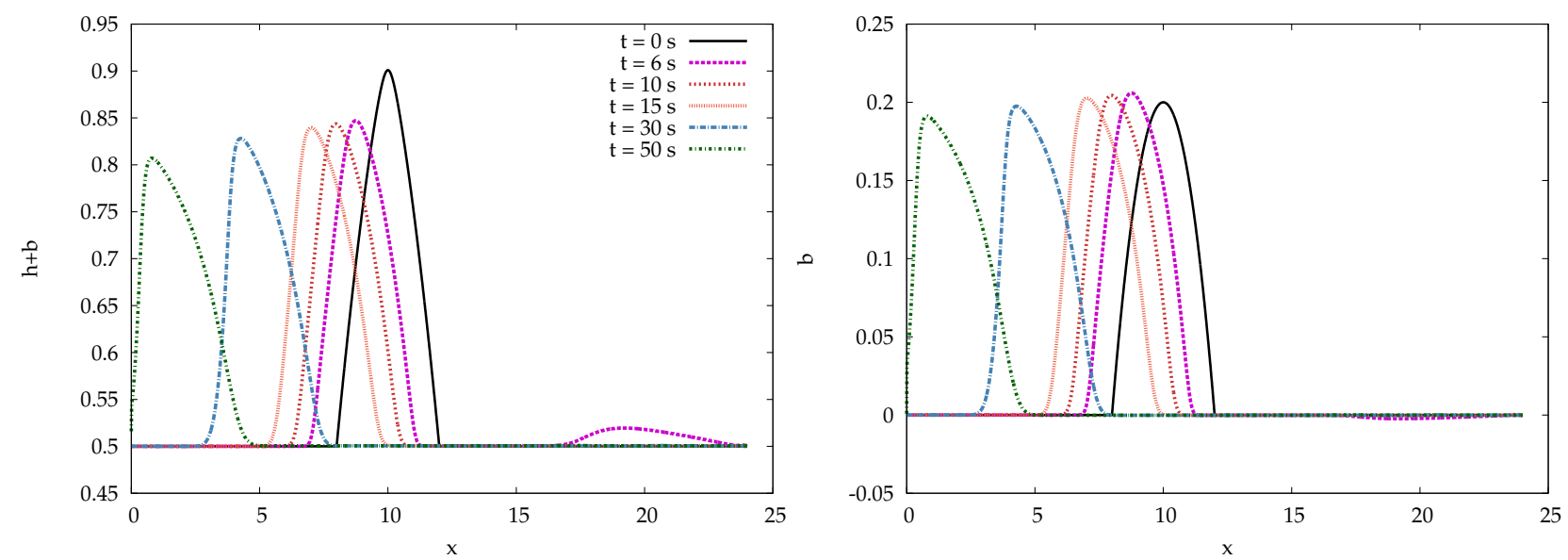

(c) ARS with Nickalls bounds

Figure 4 - Anti-dune: Comparison of anti-dune evolution for different schemes at different times.

In fact, we begin by solving the shallow water system by imposing $A_{g}=0$ in order to obtain a steady state solution; in our case, we have imposed an intermediate time equal to $20 \mathrm{~s}$. Starting from this last solution, we solve the SWExner equations by defining the sediment flux $q_{s}$ using the Grass formula 


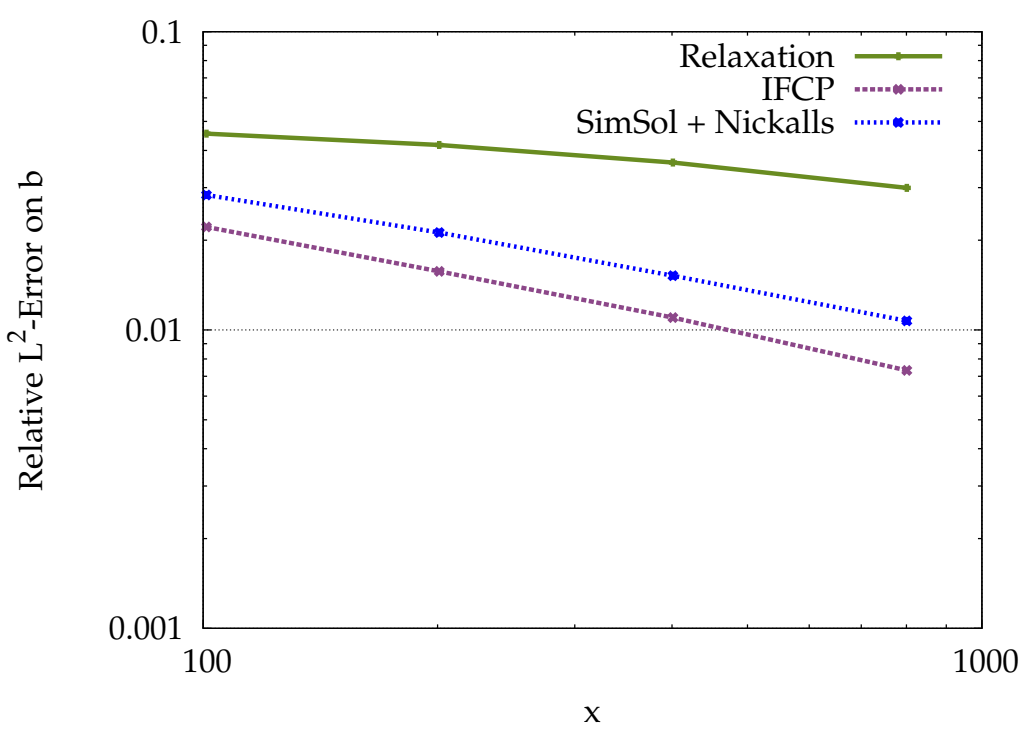

Figure 5 - Antidune: Relative $L^{2}$-error on $b$.

with $A_{g}=0.0005$ and $m=3$. With a 1000-element mesh, the numerical solutions at time $T=15$ are reported on fig. 6. It has been shown in [13] that a usual splitting strategy based on an upwind discretization of the Exner equation introduces instabilities in the numerical solution for this test case. These spurious oscillations result from the fact that the flow partly reaches a torrential regime. A possible explanation results from the fact that using the (both positive !) eigenvalues associated with the SW system implies a lost of information related to the negative wave velocity. Here, we do not observe instabilities and we observe an erosion of the dune which mainly operates at the downstream of its top; the lost materials are evacuated at the downstream of the flow. As for the other test cases, one notes that the relaxation model returns a quite diffusive solution compared to the ones obtained with the ARS and the IFCP schemes which both return very similar solutions. The fig. 7 shows that both ARS and IFCP schemes are first order accurate for this test case.
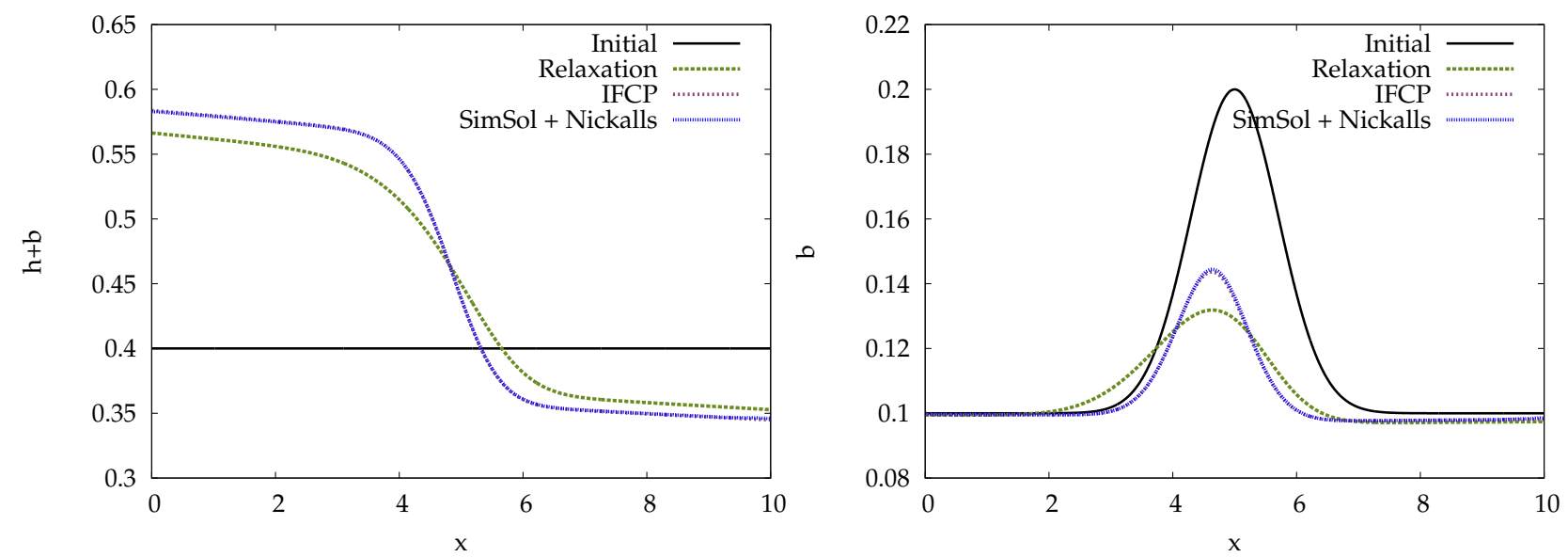

Figure 6 - Trancritical flow without shock: Comparison of dune evolution for different numerical schemes. 


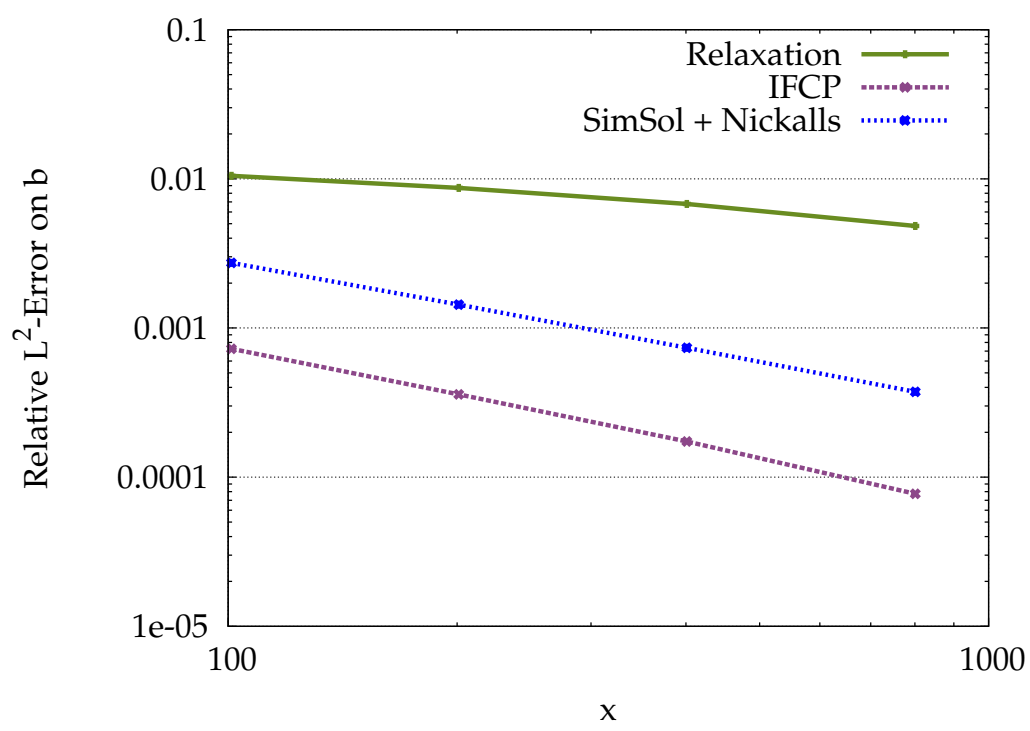

Figure 7 - Trancritical flow without shock: Relative $L^{2}$-error.

\subsection{Dam break over a wet bottom topography.}

For this test case, we consider a dam break over a flat wet bottom. We define the Exner law with the Grass formula with parameters $A_{g}=0.005$ and $m=3$. The channel is $10 \mathrm{~m}$ long and we impose the following initial conditions,

$$
\begin{cases}h(0, x)= \begin{cases}2 m, & \text { if } x \leqslant 5 m \\ 0.125 m, & \text { if } x>5 m,\end{cases} \\ u(0, x)=0 \mathrm{~m} / \mathrm{s}, \\ b(0, x)=0 \mathrm{~m} .\end{cases}
$$

The results on fig. 8 are obtained with a 1000-element mesh at time $T=1 \mathrm{~s}$. This test case has been presented in [2]. As for the previous test case, a classical splitting strategy fails to reproduce the phenomenon since numerical instabilities grow with time. Here also, the use of one step solvers cure this problem. For this test case, the results are quite similar for the three schemes. The error curves presented on fig. 9 show that the IFCP scheme is the most accurate in this case and the first order accuracy is not achieved due to the presence of the shock wave in the solution.

\subsection{Dam break over a dry bottom topography.}

The test case is the same as the previous one excepted that the initial water height exhibits dry zone,

$$
h(0, x)= \begin{cases}2 m, & \text { if } x \leqslant 5 m, \\ 0 m, & \text { if } x>5 m .\end{cases}
$$

For this test case, we include the friction term in the model (1). Note that the relaxation solver and the IFCP one are not adapted to handle vacuum, at least in the way we computed them. Then these schemes are run with a thin layer of water on the right part of the domain $h=10^{-3} \mathrm{~m}$. The results in fig. 10 are obtained with a 1000-element mesh at time $T=1 \mathrm{~s}$. The three solutions are free of oscillations and exhibit similar features even if there are noticeable differences in the eroded region, i.e. at the location of the initial dam break, see fig. 10. It is worth underlying that if we start from vacuum on the right, one needs to take a low bound for the time step (here, we have set a CFL condition of 0.1 ) to ensure the whole stability of the solver. Considering a small layer of water on 

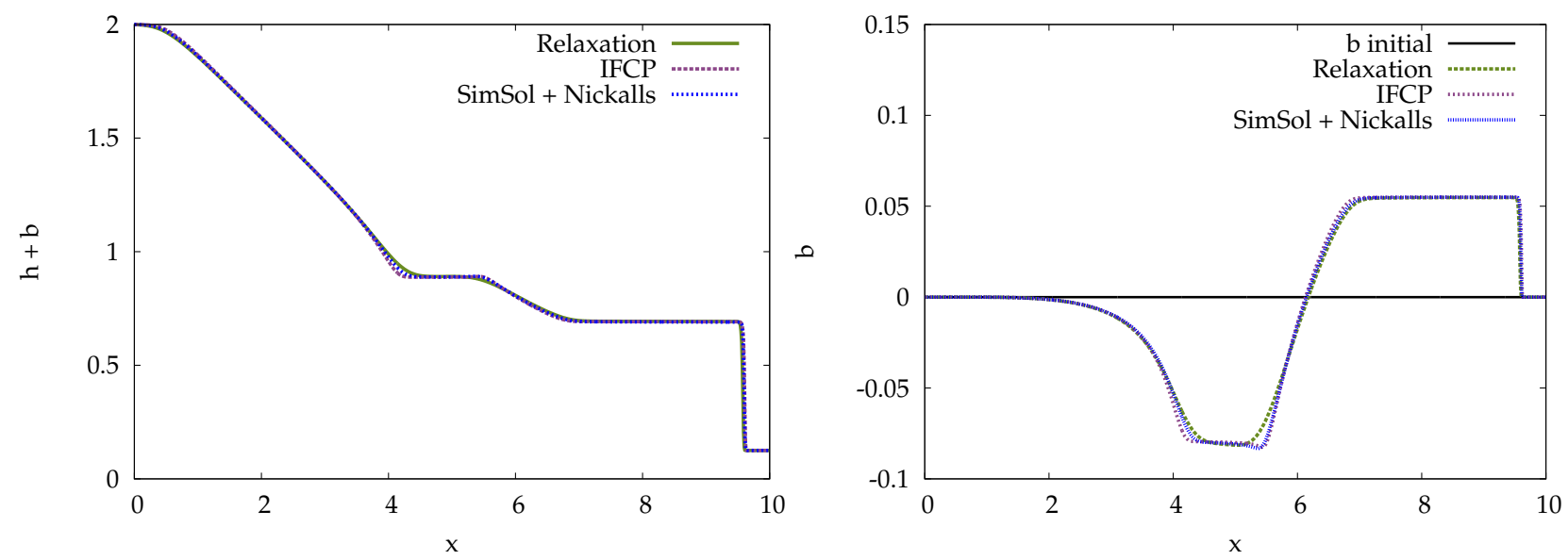

Figure 8 - Dam break over a wet bottom: Free surfaces (left) and bottom topographies (right) for different schemes.

the right part of the dam, as it is done for the two other schemes, allows to recover classical CFL condition of order 1. Due to the reasons previously mentioned in the case of a wet bottom, the error curves in fig. 11 do not show a first order accuracy and here again the IFCP scheme is the most accurate.

\section{$5 \quad$ Stabilization of splitting strategies}

In the first part of this work, we introduced a new one step solver for the SWExner system (1). Its main interest is to propose a very simple implementation and important stability (water depth positivity) and accuracy (preservation of equilibria) properties. In this section, we propose to investigate the other way to design numerical solver for the SWExner system by considering the splitting approach. Indeed, we begin by exhibiting that our ARS scheme can be understood as a stable splitting scheme, and then we extend this result to generic solvers, as the ones that are implemented in the industrial softwares. We illustrate our purpose by several numerical results.

As mentioned in the introduction the splitting strategy has been proved to be unstable in many recent works $[10,29,28,13,2]$ that have motivated the introduction of one-step methods. But all these studies consider the same kind of splitting method where fluid and solid softwares only share data as input for the initialization of the next time step. We will show in this section that if, in addition, one accepts to modify a single parameter from the fluid solver - namely the wave speed for ARS type solvers - by a value that is computed from the solid solver, it is enough to stabilize the whole method. The proposed technic is different from the one introduced in $[43,45,30]$ and referred to as the weak coupling approach since the authors propose to modify the numerical discretization in the solid solver. Up to our knowledge, the method we propose in this paper is the first one for which one can prove stability properties. This is mainly related to the link that can be made with one step solvers.

Le us now come back to the ARS introduced in Section 3 as a one-step solver and show that it can also be interpreted as a splitting strategy. Indeed, starting from the vectors $\left(h^{n}, q^{n}, b^{n}\right)$ that are shared by the two solvers, it can be divided in five steps,

- Computation of the wave velocities by using formula (3.19)-(3.20). This step is performed 


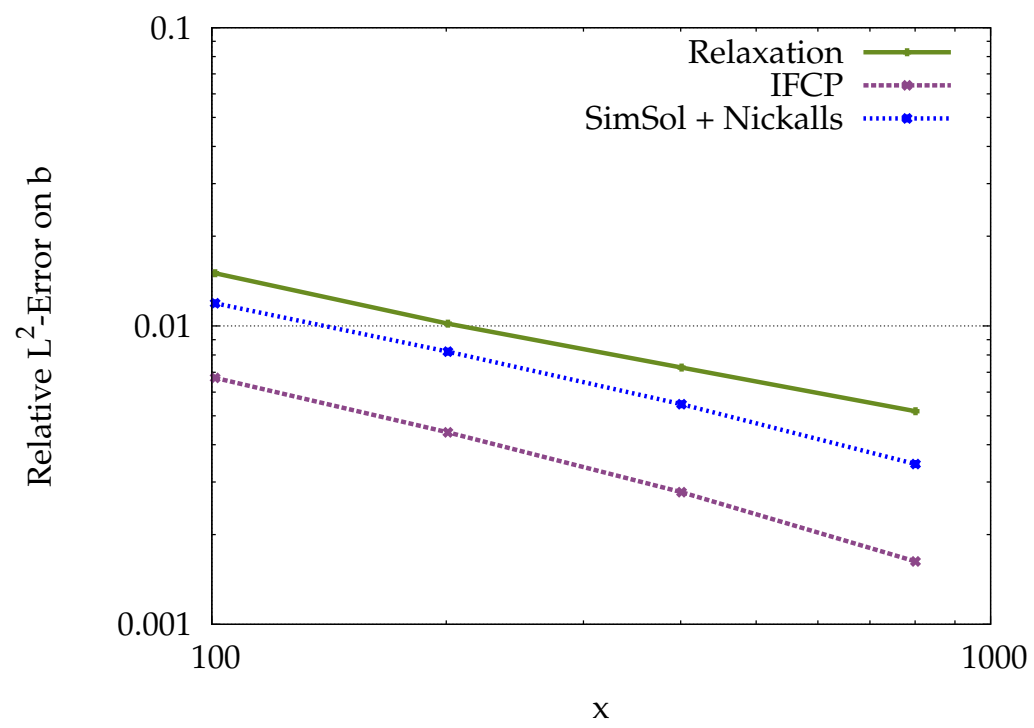

Figure 9 - Dam break over a wet bottom: Relative $L^{2}$-error.
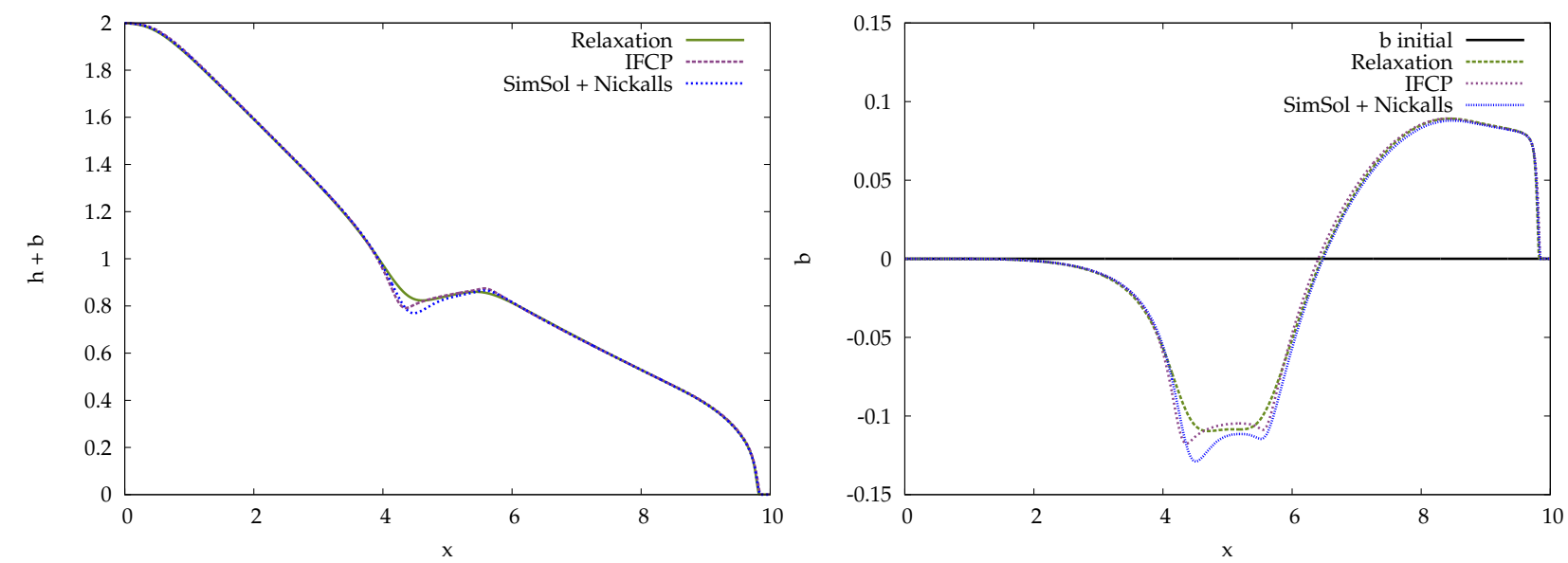

Figure 10 - Dam break over a dry bottom: Free surfaces (left) and bottom topographies (right) for different schemes.

by the solid solver since it depends on the sediment flux formula $q_{s}(h, u, b)$.

- Update of the bottom topography by computing intermediate values with formula (3.8)(3.9) and value of the new bottom topography $b^{n+1}$ with formula (3.14). This step is also performed by the solid solver since it also depends on the sediment flux formula $q_{s}(h, q, b)$.

- Transmission of wave velocities and new bottom topography to the fluid solver.

- Update of the fluid quantities by using ARS described in Section 3 that is nothing but ARS introduced in [3] with bottom topography and wave velocities given by the solid solver. Here also the computation of the new values $h^{n+1}$ and $q^{n+1}$ uses (3.14). The computation of the new discharge taking into account the friction term is included in this step.

- Transmission of new fluid quantities to the solid solver.

Since steps one and two on the first hand and step four on the other hand are now independent, it is possible to modify their numerical treatment separately. In the following, we compare three different numerical strategies, 


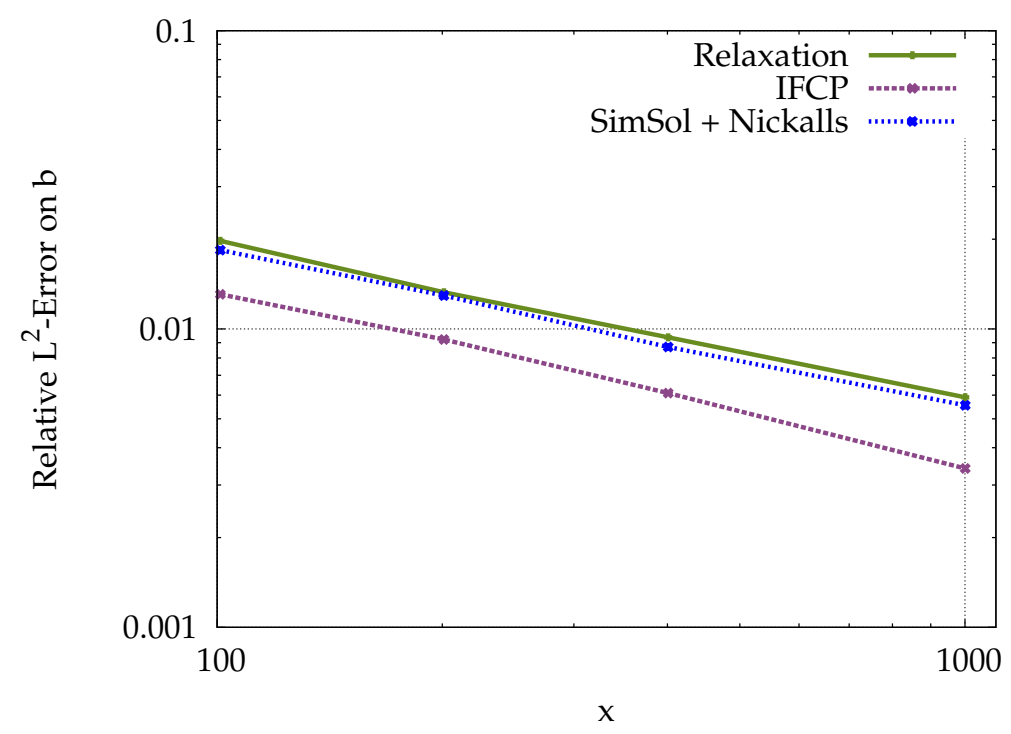

Figure 11 - Dam break over a dry bottom: Relative $L^{2}$-error.

- ARS presented in Section 3.

- Step two is replaced by a simple centered finite difference discretization for the Exner equation, i.e. what is classically done in industrial software for the solid part.

- Step two is replaced by a simple centered finite difference discretization for the Exner equation AND Step four is performed by using wave velocities with $q_{s}=0$, i.e. wave velocities of the classical shallow water equations, see Subsection 3.3. This numerical strategy is the one that is implemented in most of the industrial softwares.

We consider the same test cases as before. We exhibit in fig. 12-16 that the modification on Step two has no consequence on the stability of the method whereas the modification on Step four leads to numerical unstabilities in all configurations, except for the dune transport in a fluvial flow. Moreover, results obtained with the two first strategies are very similar.
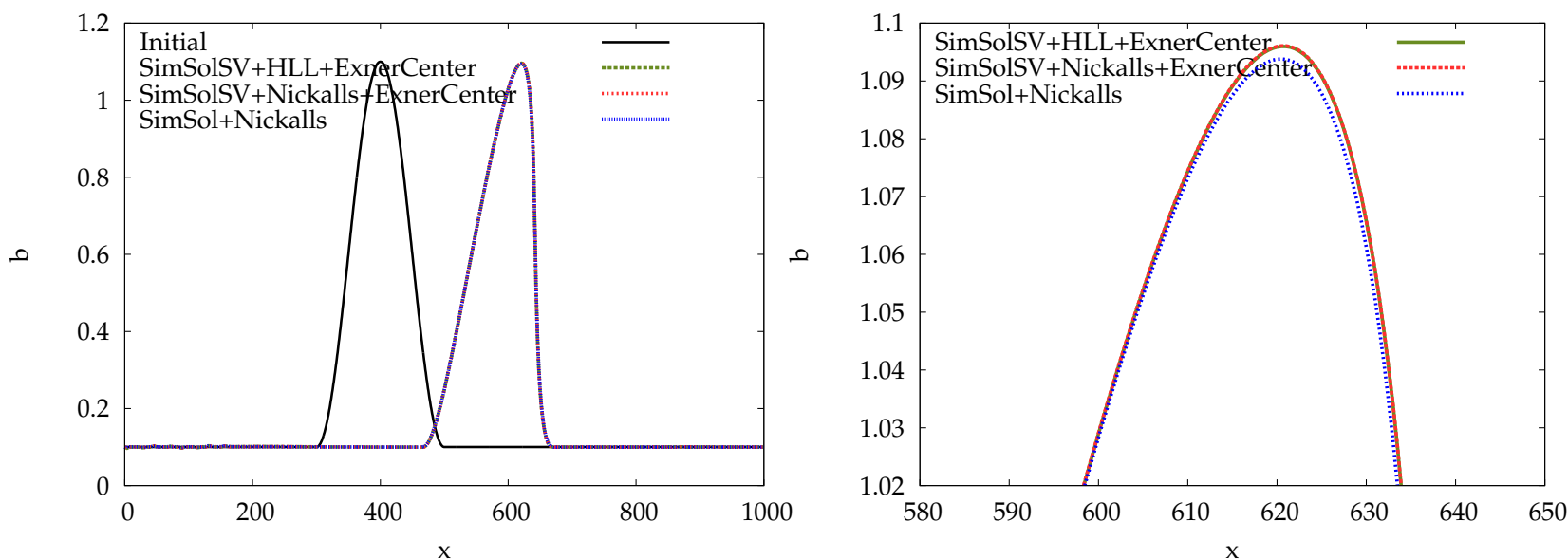

Figure 12 - Fluvial flow: Comparison of dune evolution for different numerical schemes. 

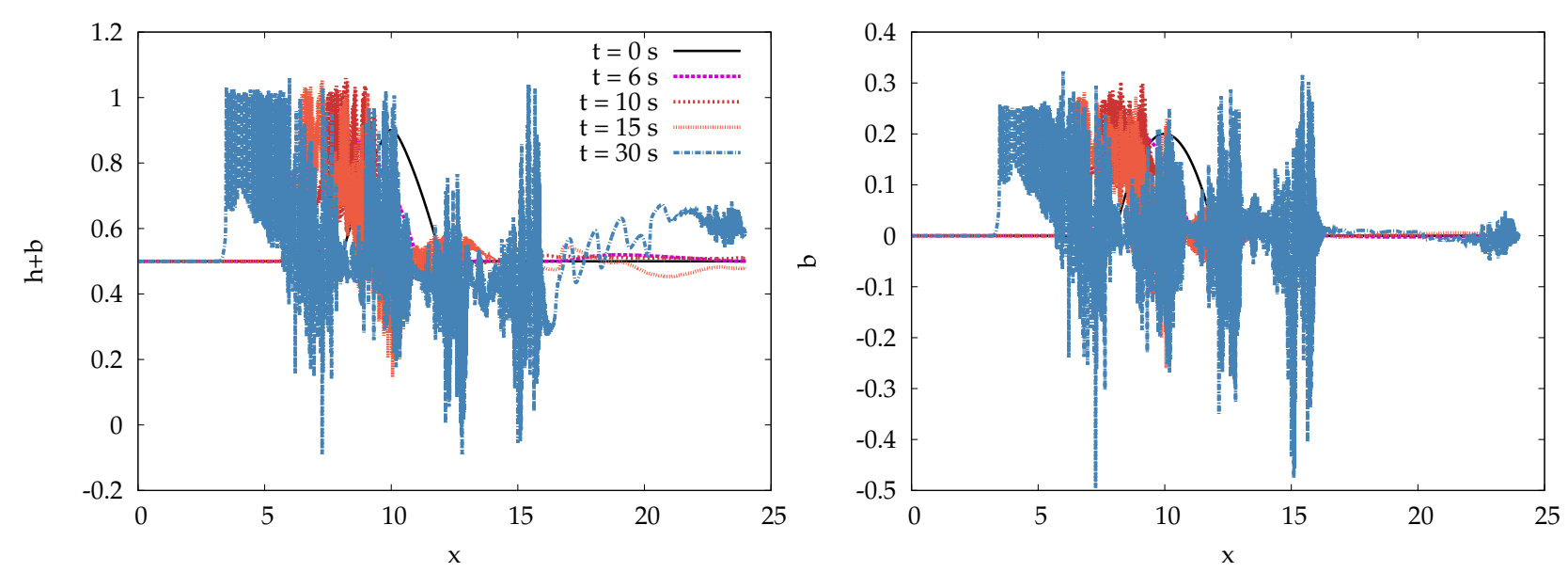

(a) SimSolSV + HLL + Exner Center
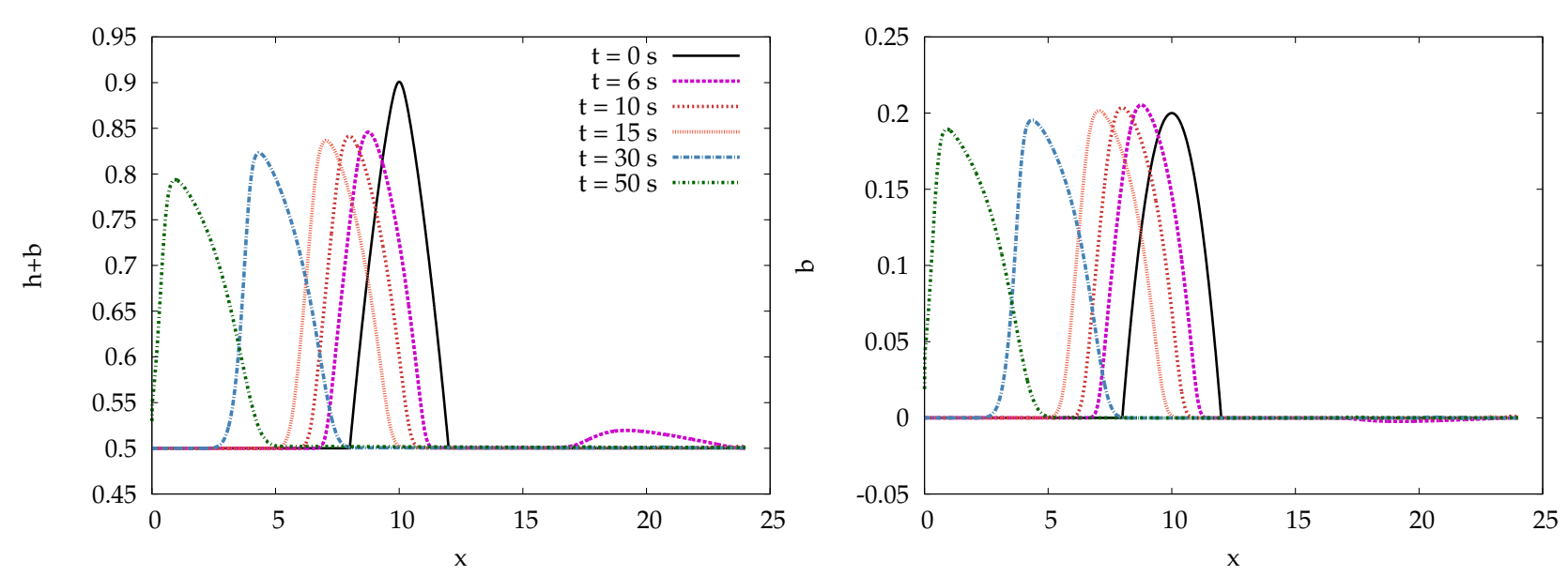

(b) SimSolSV + Nickalls + Exner Center
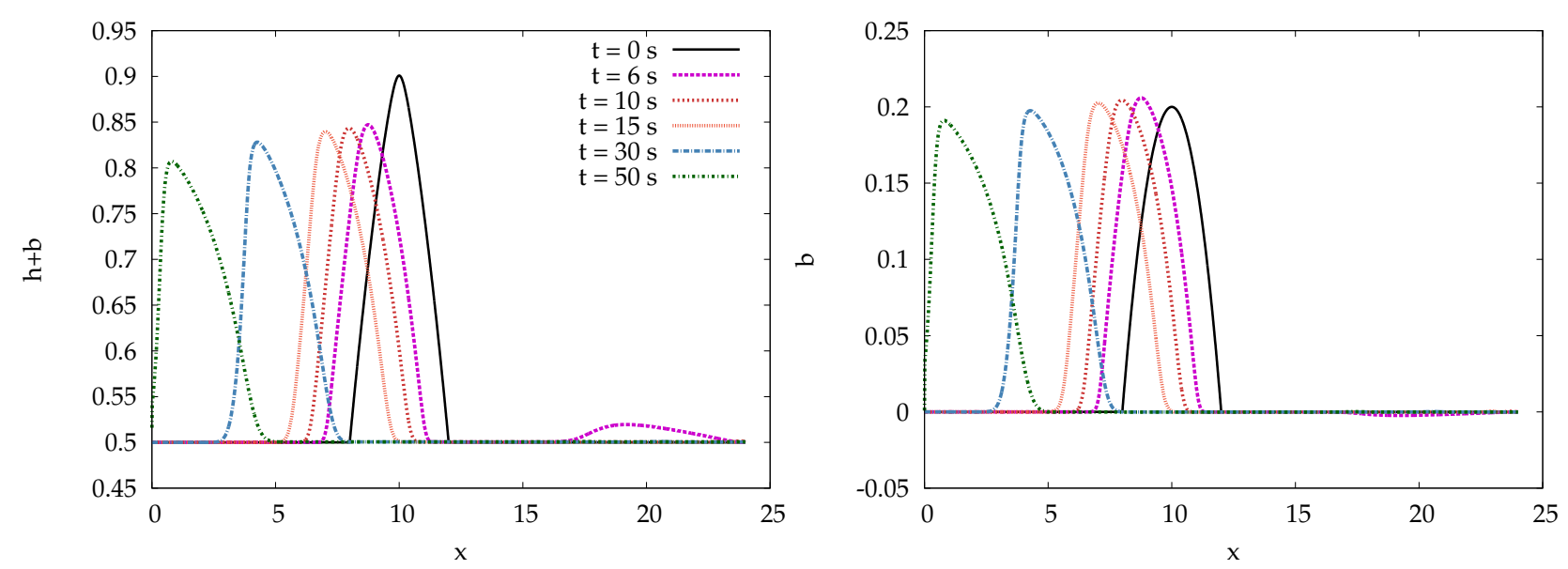

(c) SimSol+Nickalls

Figure 13 - Torrential flow: Comparison of dune evolution for different splitting strategies. 

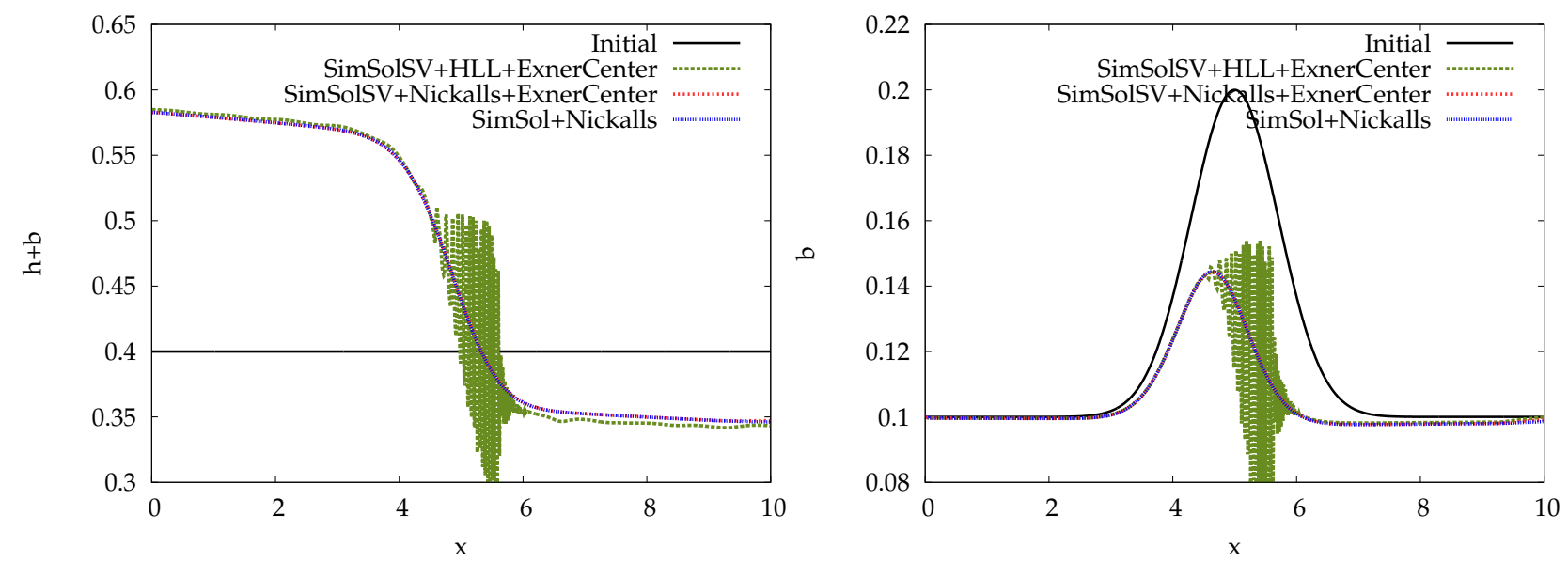

Figure 14 - Transcritical flow without shock: Comparison of dune evolution for different splitting strategies.
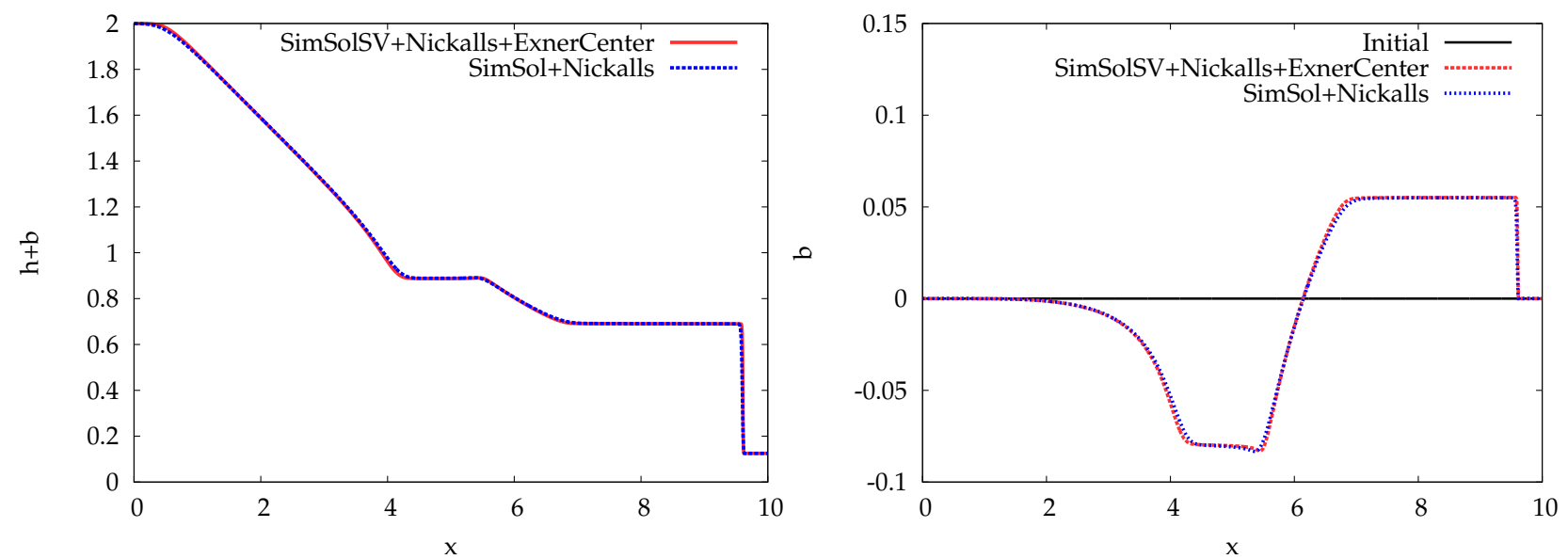

Figure 15 - Dam break over a wet bottom: Free surfaces (left) and bottom topographies (right) for different splitting strategies.
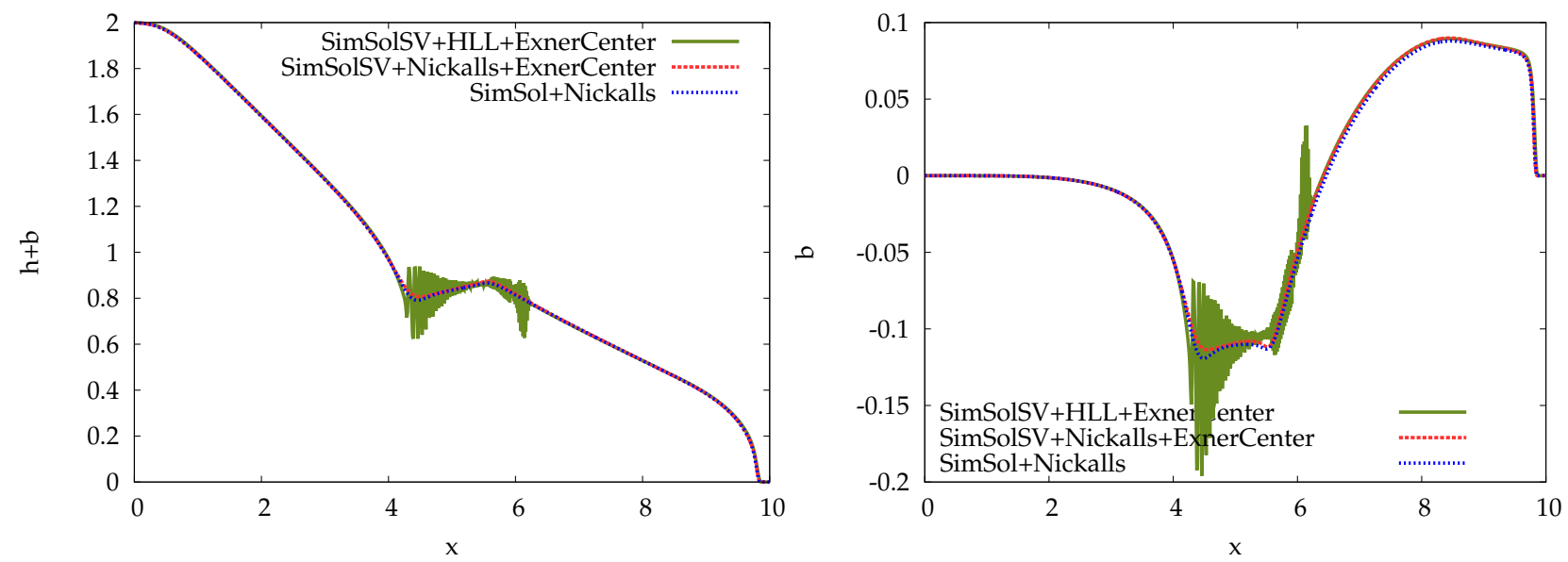

Figure 16 - Dam break over a dry bottom: Free surfaces (left) and bottom topographies (right) for different splitting strategies. 


\section{Conclusion.}

We have introduced a Godunov-type method based on a new Approximate Riemann Solver for the SWExner system (1). The scheme avoids using an approximate Jacobian matrix of the system, has been proved to be positive and well-balanced for various physically relevant equilibria and has been successfully tested on two families of discriminant numerical test cases.

Moreover this ARS scheme can be interpreted as a stable splitting strategy for solving the SWExner system. Thus it helps to understand the requirements to stabilize the splitting approach that is used in the industrial softwares. In particular we exhibit that a slight modification of the splitting approach, that only consists in modifying the wave velocities in the fluid solver, leads to stable simulations for all the test cases we performed; hence, it constitutes the first splitting approach with demonstrated stability properties.

Acknowledgements. P. U. would like to thank AMIES and EDF for their financial support.

\section{References}

[1] C. Ancey, A. C. Davidson, T. Böhm, M. Jodeau, and P. Frey. Entrainment and motion of coarse particles in a shallow water stream down a steep slope. J. Fluid Mech., 595:83-114, 2008.

[2] E. Audusse, C. Berthon, C. Chalons, O. Delestre, N. Goutal, M. Jodeau, J. Sainte-Marie, J. Giesselmann, and G. Sadaka. Sediment transport modelling: relaxation schemes for Saint-Venant-Exner and three layer models. In EDP Sciences, editor, ESAIM:Proc., pages 80-94, 2012.

[3] E. Audusse, C. Chalons, and P. Ung. A simple well-balanced and positive numerical scheme for the shallow-water system. Commun. Math. Sci., 13(5):1317-1332, 2015.

[4] E. Audusse, O. Delestre, M. H. Le, M. Masson-Fauchier, P. Navaro, and R. Serra. Parallelization of a relaxation scheme modelling the bedload transport of sediments in shallow water flow. In EDP Sciences, editor, ESAIM: Proc., pages 80-94, 2013.

[5] F. Benkhaldoun, S. Sahmim, and M. Seaid. Solution of the sediment transport equations using a finite volume method based on sign matrix. SIAM J. Sci. Comput., 31(4):2866-2889, 2009.

[6] F. Benkhaldoun, S. Sahmim, and M. Seaid. Mathematical development and verification of a finite volume model for morphodynamic flow applications. Adv. App. Math. Mech., 3(4):470-492, 2011.

[7] F. Bouchut. Nonlinear stability of finite volume methods for hyperbolic conservation laws and well-balanced schemes for sources. Birkhäuser Verlag, Frontiers in Mathematics, 2004.

[8] A. Bouharguane and B. Mohammadi. Minimization principles for the evolution of a soft sea bed interacting with a shallow sea. Int. J. Comput. Fluid D., 26:163-172, 2012.

[9] A. Canestrelli, A. Siviglia, M. Dumbser, and E.F. Toro. Well-balanced high-order centred schemes for nonconservative hyperbolic systems. Applications to shallow water equations with fixed and mobile bed. Adv. Water Resour., 32:834-844, 2009.

[10] Z. Cao, R. Day, and S. Egashira. Coupled and decoupled numerical modeling of flow and morphological evolution in alluvial rivers. J. Hydr. Res., 128:306-321, 2002.

[11] M. J. Castro Díaz, E. D. Fernández-Nieto, and A. M. Ferreiro. Sediment transport models in shallow water equations and numerical approach by high order finite volume methods. Comput. \&f Fluids, 37:299-316, 2008.

[12] S. E. Coleman and V. I. Nikora. Exner equation: A continuum approximation of a discrete granular system. Water Resour. Res., 45, 2009.

[13] S. Cordier, M. H. Le, and T. Morales de Luna. Bedload transport in shallow water models: Why splitting (may) fail, how hyperbolicity (can) help. Adv. Water Resour., 34:980-989, 2011.

[14] O. Delestre. Simulation du ruissellement d'eau de pluie sur des surfaces agricoles. PhD thesis, Université d'Orl'eans, 2010.

[15] A. I. Delis and I. Papoglou. Relaxation approximation to bed-load sediment transport. J. Comp. Ap M., 213:521546, 2008.

[16] F. M. Exner. Über die wechselwirkung zwischen wasser und geschiebe in flüssen. Akad. Wiss. Wien Math. Naturwiss. Klasse, 134(2a):165-204, 1925.

[17] E. D. Fernández-Nieto, M. J. Castro Díaz, and C. Parés. On an Intermediate Field Capturing Riemann Solver Based on a Parabolic Viscosity Matrix for the Two-Layer Shallow Water System. J. Sc. Comput., 48:117-140, 2011. 
[18] E. D. Fernández-Nieto, C. Lucas, T. Morales de Luna, and S. Cordier. On the influence of the thickness of the sediment moving layer in the definition of the bedload transport formula in Exner systems. Comput. Fluids, 91:87-106, 2014.

[19] E. D. Fernández-Nieto, T. Morales de Luna, Narbona-Reina G., and J. Zabsonré. Formal deduction of the saintvenant-exner model including arbitrarily sloping sediment beds and associated energy. M2AN, 2016.

[20] G. Gallice. Solveurs simples positifs et entropiques pour les systèmes hyperboliques avec terme source. $C$. $R$. Math. Acad. Sci. Paris, 334(8):713-716, 2002.

[21] G. Gallice. Positive and entropy stable Godunov-type schemes for gas dynamics and MHD equations in Lagrangian or Eulerian coordinates. Num. Math., 94(4):673-713, 2003.

[22] G. Garegnani, G. Rosatti, and L. Bonaventura. Free surface flows over mobile bed: mathematical analysis and numerical modeling of coupled and decoupled approaches. Commun. Appl. Ind. Math., 2(1), 2011.

[23] G. Garegnani, G. Rosatti, and L. Bonaventura. On the range of validity of the Exner-based models for mobile-bed river flow simulations. J. Hydraul. Res., 2013.

[24] J.-F. Gerbeau and B. Perthame. Derivation of Viscous Saint-Venant System for Laminar Shallow Water. Discrete Cont. Dyn. Syst. Ser. B, 1(1), 2001.

[25] A. J. Grass. Sediment transport by waves and currents. SERC London Cent. Mar. Technol., FL29, 1981.

[26] P. H. Gunawan, R. Eymard, and S. R. Pudjaprasetya. Staggered scheme for the Exner-shallow water equations. Computat. Geosci., 2015.

[27] A. Harten, P.-D. Lax, and B. van Leer. On upstream differencing and Godunov-type schemes for hyperbolic conservation laws. SIAM Review, 25(1):53-61, 1983.

[28] J. Hudson, J. Damgaard, N. Dodd, T. Chesher, and A. Cooper. Numerical approaches for 1D morphodynamic modelling. Coast. Eng., 52:691-707, 2005.

[29] J. Hudson and P. K. Sweby. Formulations for numerically approximating hyperbolic systems governing sediment transport. J. Sci. Comput., 19:225-252, 2003.

[30] C. Juez, J. Murillo, and García-Navarro P. A 2d weakly-coupled and efficient numerical model for transient shallow flow and movable bed. Adv. Water Resour., 71:93-109, 2014.

[31] R.J. Leveque. Finite Volume Methods for Hyperbolic Problems. Cambridge University Press, 2002.

[32] Logiciel DELFT3D. http://oss.deltares.nl/web/delft3d.

[33] Logiciel HEC-RAS. http://www.hec.usace.army.mil/software/hec-ras.

[34] Logiciel MIKE. http://www.mikebydhi.com.

[35] Logiciel TELEMAC-MASCARET. http://www.opentelemac.org.

[36] J. Murillo and P. Garcia-Navarro. An Exner-based coupled model for two-dimensional transient flow over erodible bed. J. Comput. Ph., 229:8704-8732, 2010.

[37] C. T. Newton. An experimental investigation of bed degradation in an open channel. Technical report, Boston Society of Civil Engineers, 1951.

[38] R. W. D. Nickalls. A new bound for polynomials when all roots are real. The Mathematical Gazette, 95(534):520526, November 2011. www.nickalls.org/dick/papers/maths/bounds2011.pdf.

[39] C. Paola and V. R. Voller. A generalized Exner equation for sediment mass balance. J. Geophys. Res., $110,2005$.

[40] A. Paquier. Modélisation et simulation de la propagation de l'onde de rupture de barrage. PhD thesis, Université Jean Monnet, October 1995.

[41] C. Parés. Numerical methods for nonconservative hyperbolic systems: a theoretical framework. SIAM J. Numer. Anal., 44(1):300-321, 2006.

[42] G. Parker. 1D Sediment Transport Morphodynamics with applications to Rivers and Turbidity Currents. http://hydrolab.illinois.edu/people/parkerg/powerpoint_lectures.htm, 2004.

[43] M. Postacchini, M. Brocchini, A. Mancinelli, and M. Landon. A multi-purpose, intra-wave, shallow water hydromorphodynamic solver. Adv. Water Resour., 38:13-26, 2012.

[44] G. Rosatti, J. Murillo, and L. Fraccarollo. Generalized Roe schemes for 1D two-phase, free-surface flows over a mobile bed. J. Comput. Ph., 227:10058-10077, 2008.

[45] A. Serrano-Pacheco, J. Murillo, and García-Navarro P. Finite volumes for 2d shallow-water flow with bed-load transport on unstructured grids. J. Hyd. Res., 50:154-163, 2012.

[46] S Soares-Frazao and Y Zech. HLLC scheme with novel wave-speed estimators appropriate for two-dimensional shallow-water flow on erodible bed. Int. J. Numer. Meth. Fl., 66:1019-1036, 2011.

[47] J. P. Soni. Laboratory study of aggradation in alluvial channels. J. Hydrol., 49:87-106, 1981.

[48] L. C. van Rijn. Sediment Transport, Part I: Bed Load Transport. J. Hydraul. Eng., 110(10):1431-1456, 1984.

[49] J. Zabsonré, C. Lucas, and E. D. Fernández-Nieto. An energetically consistent viscous sedimentation model. Math. Models Methods Appl. Sci., 19(3):477-499, 2009. 